\title{
Mitogen-Activated Protein Kinase Phosphatase-1 Is a Key Regulator of Hypoxia-Induced Vascular Endothelial Growth Factor Expression and Vessel Density in Lung
}

Kristin M. Shields, ${ }^{*}$ Evgeniy Panzhinskiy, ${ }^{\dagger}$ Nana Burns, ${ }^{*}$ W. Michael Zawada, ${ }^{\dagger}$ and Mita Das $^{\dagger}$

From the Department of Pediatrics, * University of Colorado Denver, Aurora, Colorado; and the Division of Pharmaceutical Sciences, ${ }^{\dagger}$ School of Pharmacy, University of Wyoming, Laramie, Wyoming

\begin{abstract}
Although mitogen-activated protein kinase phosphatase-1 (MKP-1) is a key deactivator of MAP kinases, known effectors of lung vessel formation, whether it plays a role in the expression of proangiogenic vascular endothelial growth factor (VEGF) in hypoxic lung is unknown. We therefore hypothesized that MKP-1 is a crucial modulator of hypoxia-stimulated vessel development by regulating lung VEGF levels. Wild-type MKP-1 ${ }^{+/+}$, heterozygous MKP-1 ${ }^{+/-}$, and deficient MKP-1 ${ }^{-/-}$mice were exposed to sea level (SL), Denver altitude (DA) (1609 $\mathrm{m}$ [5280 feet]), and severe high altitude (HYP) $(\sim 5182 \mathrm{~m}[\sim 17,000$ feet $])$ for 6 weeks. Hypoxia enhanced phosphorylation of $\mathbf{p 3 8}$ MAP kinase, a substrate of MKP-1, as well as $\alpha$ smooth muscle actin ( $\alpha$ SMA) expression in vessels, respiratory epithelium, and interstitium of phosphatase-deficient lung. $\alpha$ SMA-positive vessel $(<50 \mu \mathrm{m}$ outside diameter) densities were markedly reduced, whereas vessel wall thickness was increased in hypoxic MKP$1^{-/-}$lung. Mouse embryonic fibroblasts (MEFs) of all three genotypes were isolated to pinpoint the mechanism involved in hypoxia-induced vascular abnormalities of MKP-1 ${ }^{-/-}$lung. Sustained phosphorylation of p38 MAP kinase was observed in MKP-1-null MEFs in response to hypoxia exposure. Although hypoxia up-regulated VEGF levels in MKP-1 ${ }^{+/+}$MEFs eightfold, only a 70\% increase in VEGF expression was observed in MKP-1-deficient cells. Therefore, our data strongly suggest that MKP-1 might be the key regulator of vascular densities through the regulation of VEGF levels in hypoxic lung. (Am J Pathol 2011, 178: 98-109; DOI: 10.1016/j.ajpath.2010.11.025)
\end{abstract}

Dual-specificity phosphatases are the key regulators of dephosphorylation of the ERK1/2, JNK $1 / 2$, and $\mathrm{p} 38$ mitogen-activated protein (MAP) kinases, and so have been designated as MAP kinase phosphatases (MKPs). MKP-1 is a member of this phosphatase family. ${ }^{1}$ MKP-1 expression in the lung is considerably higher than in other tissues, and it has been shown to protect arteries from a proinflammatory state by suppressing the activities of p38 and JNK MAP kinases in the vascular endothelium. ${ }^{2,3}$ Although MKP-1 is a hypoxia-inducible phosphatase, and hypoxia triggers angiogenesis, ${ }^{4,5}$ the role of MKP-1 in the formation and maintenance of vascular network in hypoxic lung remains unexplored.

Vascular endothelial growth factor (VEGF) is crucial for the formation of new blood vessels and plays a central role in the development and maturation of a healthy vasculature, as well as in vascular pathophysiological conditions. ${ }^{5}$ Chronic hypoxia exposure leads to increased VEGF expression in the lung and subsequent angiogenesis. ${ }^{6-8}$ Recent studies have demonstrated that VEGF receptor pathway induces $\mathrm{MKP}-1$ transcription in endothelial cells ${ }^{9,10}$; however, the role of MKP-1 in hypoxiainduced VEGF expression in the lung is unknown.

Although MKP-1-deficient mice seem to be largely normal, these animals have an exaggerated innate immune response to lipopolysaccharide (LPS) and exhibit increased serum levels of various cytokines, including tumor necrosis factor- $\alpha$ (TNF- $\alpha)$, interleukin- 6 (IL-6), interferon- $\gamma, \mathrm{IL}-10, \mathrm{IL}-12$, and monocyte chemotactic protein-1 (MCP-1; also known as C-C motif chemokine 2 protein, or CCL2). ${ }^{11-13}$ MKP-1-null mice are

\footnotetext{
Supported by NIH grant HL64917 (M.D.)

Accepted for publication September 17, 2010.

Address reprint requests to Mita Das, Ph.D., School of Pharmacy, University of Wyoming, 1000 E. University Ave., Laramie, WY 82071 E-mail: mita.das5000@gmail.com.
} 
lean and resistant to diet-induced obesity. ${ }^{14}$ Activation levels of ERK1/2, JNK1/2, and p38 MAP kinase are elevated in skeletal muscle and white adipose tissue of these mice. MKP-1 has also been shown to play a role in the maintenance of bone mass by negatively regulating MAP kinase-dependent osteoclast signaling. ${ }^{15}$ In LPS-treated $\mathrm{MKP}-1^{-/-}$mice, the lungs manifest edema, thickening of the alveolar septa, and infiltration by leukocytes in the interstitial space. ${ }^{13} \mathrm{Jin}$ et $\mathrm{al}^{16}$ recently reported that mice deficient in MKP-1 develop more severe pulmonary hypertension, with lower levels of nitric oxide synthase and greater arginase levels, after 4 weeks in hypoxia than do wild-type mice. However, little information exists regarding the vascular status of the lungs of MKP-1-deficient mice challenged with chronic exposure to hypoxia. Therefore, using MKP-1-deficient mice, we tested the hypothesis that MKP-1 functions as a regulator of the maintenance and development of vascular network in hypoxic lung by controlling VEGF levels.

In the present study, we found that chronic exposure to hypoxia induces exaggerated p38 MAP kinase activation and $\alpha$ SMA expression in lung of MKP-1-null mice. Most importantly, marked reduction in vessel densities and remodeling of the vascular wall were observed in lung of hypoxia-exposed MKP-1-deficient mice. Using cultured mouse embryonic fibroblasts (MEFs), we demonstrated that hypoxia-stimulated upregulation of VEGF expression is defective on deletion of the MKP-1 gene, suggesting that MKP-1 is a crucial phosphatase for the regulation of VEGF levels and vessel densities in hypoxic lung.

\section{Materials and Methods}

\section{Animal Studies}

All experiments with mice were approved by the University of Colorado Denver Institutional Animal Care and Use Committee. MKP-1 heterozygous mice were provided by Lexicon Pharmaceuticals (The Woodlands, TX). Male mice, 4 to 5 weeks old, of the three genotypes $\mathrm{MKP}_{-} 1^{+/+}, \mathrm{MKP}_{-} 1^{+/-}$, and $\mathrm{MKP}_{-} 1^{-1-}$ were divided into three groups and exposed to sea level (SL), ambient Denver (DA) (1609 m [5280 feet]), or simulated severe high altitude (HYP) (5182 m [17,000 feet]) conditions for 6 weeks, according to a previously described method. ${ }^{17,18}$ Briefly, the first group was placed in a hyperbaric chamber in which the pressure was increased to simulate sea-level barometric pressure $\left(P_{\mathrm{B}}=760 \mathrm{mmHg}\right)$. The second group was kept at Denver's altitude $\left(P_{\mathrm{B}}=630 \mathrm{mmHg}\right)$. The third group was placed in a hypobaric chamber and exposed to simulated severe high altitude $\left(P_{\mathrm{B}}=410 \mathrm{mmHg}\right)$. The chambers were continuously flushed with room air to prevent accumulation of $\mathrm{CO}_{2}, \mathrm{NH}_{3}$, and $\mathrm{H}_{2} \mathrm{O}$. Both hyperbaric and hypobaric exposures of mice were 24 hours/day, except when the chambers were opened for 10 to 15 minutes every 2 days to clean cages and replenish food and water. All mice were exposed to a
12:12 hours light-dark cycle and were allowed free access to standard chow and water. After 6 weeks, mice were anesthetized with tribromoethanol $(500 \mathrm{mg} /$ $\mathrm{kg}$, i.p.), weighed, and subjected to whole-body perfusion with EDTA-containing heparinized saline. Lungs were inflated with $1 \%$ agarose, solidified on ice, fixed in formalin overnight, embedded in paraffin, and then sectioned.

\section{Genotyping of Mouse}

For genotype analysis, DNA from mouse tail piece was isolated using a DNeasy kit (Qiagen, Valencia, CA). PCR analysis was performed using primer sets specific for MKP-1 and neomycin: MKP-1 forward: 5'-CTTCTCGGAAGGATATGCTTGACG-3', reverse: 5'-TCAGTTCAACTGAGTCTCAGTGAGG-3' and neomycin forward: 5'-GCAGCGCATCGCCTTCTATC-3', reverse: 5'GGCTCCTCCCAAGCTTTGTATATCC-3'.

\section{Isolation of Mouse Embryonic Fibroblasts}

Pregnant (11 to 13 days) female mice were sacrificed with pentobarbital (200 mg/kg i.p.) followed by cervical dislocation. The uterine horns were removed and the embryos were dissected in sterile PBS. Fetal membranes were used for genotyping, and embryos minus primordial organs were mechanically dispersed, strained, and plated on $0.1 \%$ gelatin-coated Petri dishes with Dulbecco's modified Eagle's medium/ Ham's F-12 medium (DMEM/F-12) containing 15\% fetal bovine serum. Confluent cells were detached with Accutase (Innovative Cell Technologies, San Diego, CA), counted with a hemocytometer, and plated for experiments.

\section{Immunohistochemistry}

The following antibodies were used for staining of the lung sections: $\alpha$ SMA (1:100) (Sigma-Aldrich, St. Louis, MO) ${ }^{19,20}$; phosphoP38 (1:50), phosphoJNK (1:100), and phosphoERK (1:100) (Cell Signaling Technology, Danvers, MA) 21,22; Ki-67 (1:100) (Epitomics, Burlingame, CA ${ }^{23,24}$; and VEGF (1:100) (Novus Biologicals, Littleton, CO). ${ }^{25}$ Immunohistochemical staining was performed according to our previously described method. ${ }^{26}$ Briefly, a universal avidin-biotin-peroxidase kit (Vector Laboratories, Burlingame, CA) was used for immunohistochemical staining. Peroxidase activity was visualized with diaminobenzidine (DAB) (Vector Laboratories). Sections were lightly counterstained with hematoxylin, coverslipped, and mounted. A universal negative control (DakoCytomation, Glostrup, Denmark) was used for staining control slides. Murine kidney, brain, heart, and spleen were also stained with the above-mentioned antibodies and were used as positive controls for immunohistochemical staining of lung sections. Images were captured at $\times 40$ with Spot Advanced software v4.0.6 (Diagnostic Instruments, Sterling Heights, MI) and a Nikon Eclipse E800 microscope. 


\section{Determination of Density and Wall Thickness of Lung Vasculature}

Vascular density and wall thickness in the lung of MKP$1^{+/+}, \mathrm{MKP}_{-} 1^{+/-}$, and $\mathrm{MKP}-1^{-1-}$ mice were measured according to previously described methods. ${ }^{27-29}$ Briefly, lung sections were stained with $\alpha \mathrm{SMA}$ antibody and lightly counterstained with hematoxylin. The number of $\alpha$ SMA-positive vessels [between 10 and $50 \mu \mathrm{m}$ outside diameter $(O D)$ ] and alveoli were counted in $\alpha S M A$-stained sections by an investigator who was unaware of the experimental conditions; 5 to 12 vessels were counted in each of the three lobes of lung in every animal. Vessel density was expressed as the ratio of vessels to alveoli.

For the evaluation of lung vascular wall thickness, Zeiss AxioVision software v2.05 was used. Diameter and wall thickness of muscular arteries were measured in $\alpha$ SMA-positive vessels (between 10 and $50 \mu \mathrm{m} \mathrm{OD)}$ ). Vascular wall thickness was expressed as the ratio of wall width to vessel width. Each lung section was assessed at five randomly captured fields at $\times 20$, and at least 50 vessels were analyzed per animal.

\section{Western Blot Analysis}

Cell lysates harvested from MEFs were separated by immunoblot analysis for the evaluation of hypoxia-induced phosphorylation of MAP kinases and levels of $\alpha S M A$ protein according to our previously described method. ${ }^{30}$

\section{Activation of MAP Kinases}

MEFs were exposed to hypoxic $\left(1 \% \mathrm{O}_{2}\right)$ conditions for $0,30,60$, and 120 minutes and then were harvested with cell lysis buffer. Protein concentrations were measured using a Bradford protein assay (Bio-Rad, Hercules, CA). Equal amounts of total protein from each experimental condition were separated on Bis-Tris gels (NuPage; Invitrogen, Carlsbad, CA) and were transferred onto polyvinylidene difluoride membranes, blocked with $5 \%$ nonfat milk in Tris-buffered salineTween for 1 hour at room temperature, and incubated overnight with antibodies against phosphoP38 (1:500), phosphoJNK (1:500), and phosphoERK1/2 (1:1000) (Cell Signaling Technology). After washing in Tris-buffered saline-Tween solution, membranes were incubated with the alkaline phosphatase-conjugated antirabbit IgG $(1: 10,000)$ (Upstate; Millipore, Billerica, MA) for 1 hour at room temperature. Blots were developed using Lumi-Phos reagent (Pierce Biotechnology, Rockford, IL), and exposed to film. Protein bands were quantified using $\mathrm{NIH}$ Image $\mathrm{J}$ software $\mathrm{v} 1.58 .^{30}$

\section{Expression of $\alpha S M A$}

MEFs were preincubated with inhibitors of MAP kinase pathways (U0126, SB203580, and SP600125, and with dimethyl sulfoxide as control) for 1 hour and then exposed to either normoxia or hypoxia for 24 hours. An- tibody against $\alpha$ SMA $(1: 20,000)$ (Sigma-Aldrich) was used for overnight incubation at $4^{\circ} \mathrm{C}$. Alkaline phosphatase-conjugated anti-mouse IgG 1:10,000 (Santa Cruz Biotechnology, Santa Cruz, CA) was used for 1 hour of incubation at room temperature.

\section{Proliferation Assay}

MEFs were plated in 96-well plates with $10 \%$ fetal bovine serum-containing media and then were growtharrested for 72 hours according to our previously described method. ${ }^{31}$ Quiescent cells were preincubated for 1 hour with or without inhibitors (U0126, SB203580, and SP600125, and with dimethyl sulfoxide as control) and then were exposed to either normoxia or hypoxia for 72 hours. At the end of the experimental period, proliferation was measured with CellTiter $96 \mathrm{AQ}_{\text {ueous }}$ One Solution reagent according to the manufacturer's protocol (Promega, Madison, WI).

\section{VEGF Enzyme-Linked Immunosorbent Assay}

For the quantitation of intracellular VEGF levels, growth-arrested MEFs were exposed to either normoxia or hypoxia in the presence of various antag-
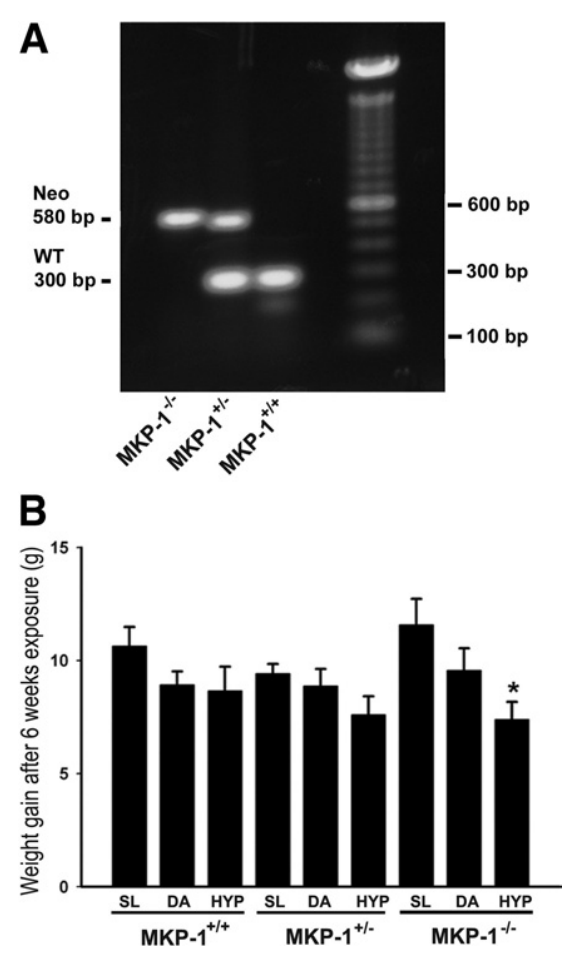

Figure 1. Chronic hypoxia exposure interferes with total body weight gain in MKP-1-deficient mice. A: Genotyping of MKP-1 $1^{+/+}, \mathrm{MKP}^{-1}{ }^{+/}$, and MKP- $1^{-/}$ mice by PCR. Mouse tail DNA amplified by PCR shows a MKP-1 band of $300 \mathrm{bp}$ and a neomycin band of $580 \mathrm{bp}$. B: Weight gain in mice in the absence of MKP-1 expression is attenuated by hypoxia exposure ( $n=8$ to 15 animals/group) $\mathrm{MKP}^{-1}{ }^{-/-}$mice had marked decrease in gain of body weight compared with MKP- $1^{+/+}$and MKP-1 $1^{+/-}$mice after 6 weeks exposure to a simulated altitude of $\sim 5182 \mathrm{~m}(\sim 17,000$ feet $) .{ }^{*} P<0.05$ compared with SL group of MKP-1-deficient mice. SL, sea level; DA, Denver altitude; HYP, high-altitude hypoxia. 
onists targeting members of the MAP kinase family for 24 hours. Cells were harvested at the end of exposure and enzyme-linked immunosorbent assay was performed with cell lysates using a mouse VEGF enzymelinked immunosorbent assay kit (RayBiotech, Norcross, GA) according to the manufacturer's protocol. Briefly, equal amounts of protein from cell lysates were added in the wells of 96-well plates, which were precoated with mouse-specific anti-VEGF antibody, incubated overnight at $4^{\circ} \mathrm{C}$, washed, and again incubated with biotinylated anti-mouse VEGF antibody for 1 hour at room temperature. After washing, horseradish peroxidase-conjugated streptavidin solution was added to each well and incubated for 45 minutes. 3,3',5,5'-tetramethylbenzidine one-step substrate reagent was added to the wells after washing and incubated for 30 minutes. Stop solution was added to the wells at the end of the incubation and plates were immediately read at $450 \mathrm{~nm}$.

\section{Statistical Analysis}

All data are expressed as arithmetic means \pm SEM. For animal studies, the sample number $n$ represents the number of mice per experimental group. For experiments with cultured cells, the $n$ represents the number of cell populations, each isolated from an individual embryo, or the number of replicate wells per test condition in representative experiments. One-way analysis of variance and then Student-Newman-Keuls multiple comparison tests were conducted for comparisons within and between groups of data points. Data were considered significantly different if $P<0.05$.

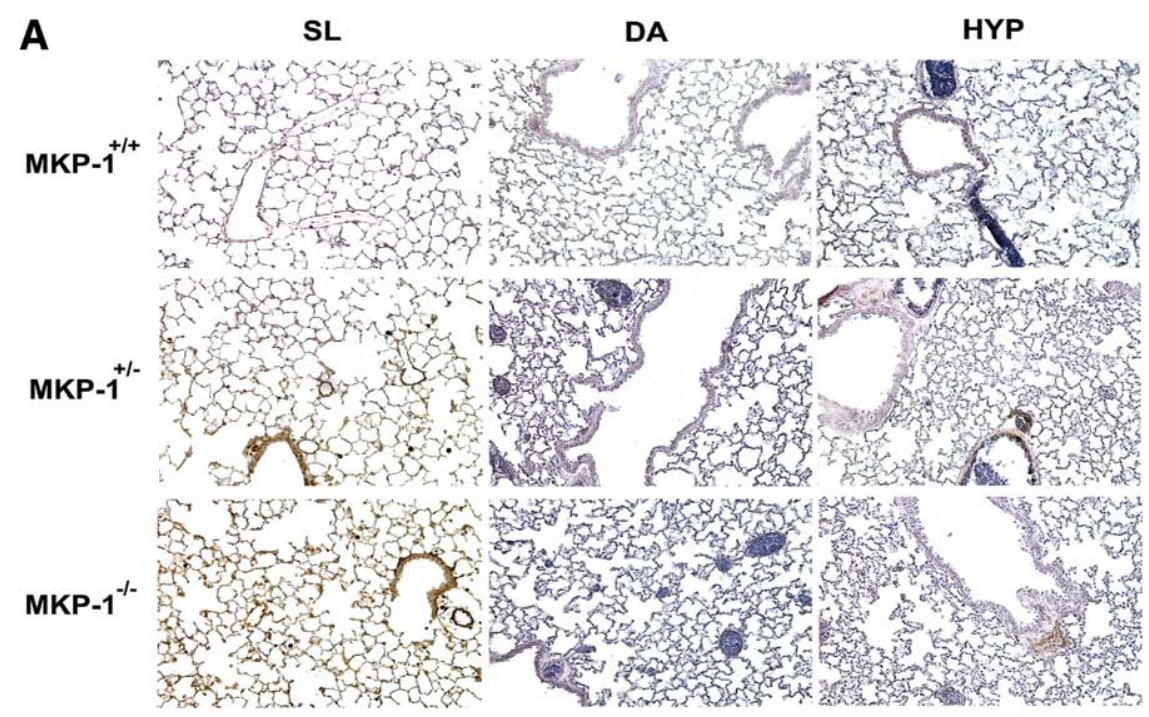

B

$\mathrm{MKP}_{-1}^{+1+}$
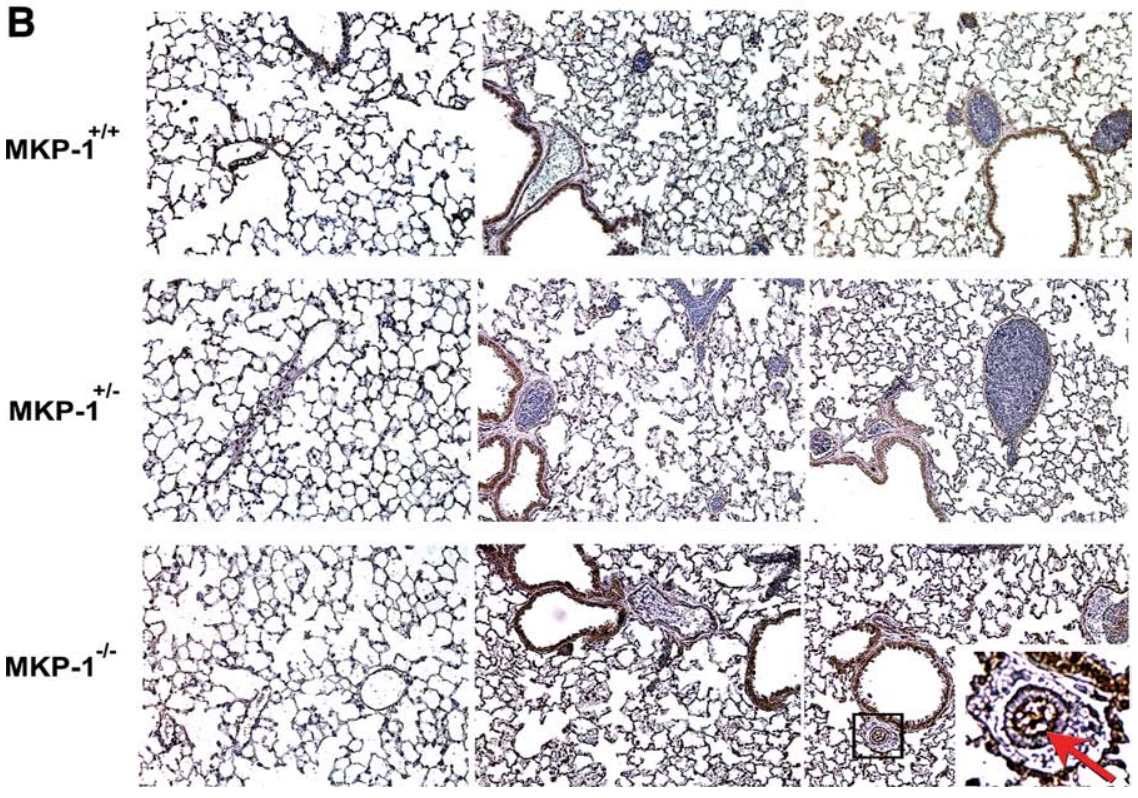

Figure 2. Heightened phosphorylation of ERK1/2 and p38 MAP kinase is observed in the lung of MKP-1-deficient mice. A: Sea-level (SL) exposed lung of MKP-1 $1^{+/-}$and MKP-1 $1^{-/-}$mice have the greatest phosphorylated ERK1/2 levels. Positive signal of immunostaining is represented as brown (DAB) in lung section $(n=3$ to 4 mice/group). B: Staining intensity of phosphoP38 MAP kinase is strongest in lung of MKP$1^{-1-}$ mice both at DA and HYP conditions $(n=$ 3 to 4 animals/group). In MKP-1 $1^{-1-}$ lung section of HYP group, the small box marks a vessel, magnified in the inset; the red arrow indicates the endothelial cell layer of the vessel. Original magnification $\times 400 ; \times 1200$ (inset) 


\section{Results}

Hypoxia Inhibits Weight Gain in MKP-1 ${ }^{-1-}$ Mice

We first genotyped $\mathrm{MKP}_{-} 1^{+/+}, \mathrm{MKP}^{-1^{+/-}}$, and MKP$1^{-I-}$ mice by PCR (Figure $1 \mathrm{~A}$ ) and exposed them to SL, DA, and HYP conditions for 6 weeks. The SL exposure was used as a control group, because mice were bred and maintained at DA (1609 m higher than SL), and this elevated altitude significantly affects the physiological responses of the animals. Hypoxia-exposed $\mathrm{MKP}-1^{-1-}$ mice had a significantly greater reduction in total body weight gain, compared with MKP-1-deficient mice in SL and DA conditions, after 6 weeks (Figure 1B). By contrast, total body weight gain was not affected by altitude in $\mathrm{MKP}-1^{+/+}$and $\mathrm{MKP}-1^{+/-}$mice (Figure 1B).
Therefore, the MKP-1 gene might be a critical regulator of body weight under hypoxic conditions.

\section{ERK1/2 and p38 MAP Kinases Are Activated in Lung of MKP-1 $1^{+/-}$and MKP-1 ${ }^{-/-}$Mice}

To evaluate the activation status of substrates of MKP-1 (ERK1/2, JNK1/2, and p38 MAP kinase), lung sections were immunostained with the antibodies against phosphoERK $1 / 2$, phosphoJNK $1 / 2$, and phosphoP38 MAP kinase. The strongest staining intensities of activated ERK $1 / 2$ were observed in the vessel wall, alveoli, and airway of SL-exposed lung of $\mathrm{MKP}^{-1^{+/}}$ and $\mathrm{MKP}-1^{-1-}$ mice (Figure 2A). There was no positive staining of phosphorylated ERK $1 / 2$ in MKP-1 ${ }^{+/+}$lung at
A

SL

DA

HYP

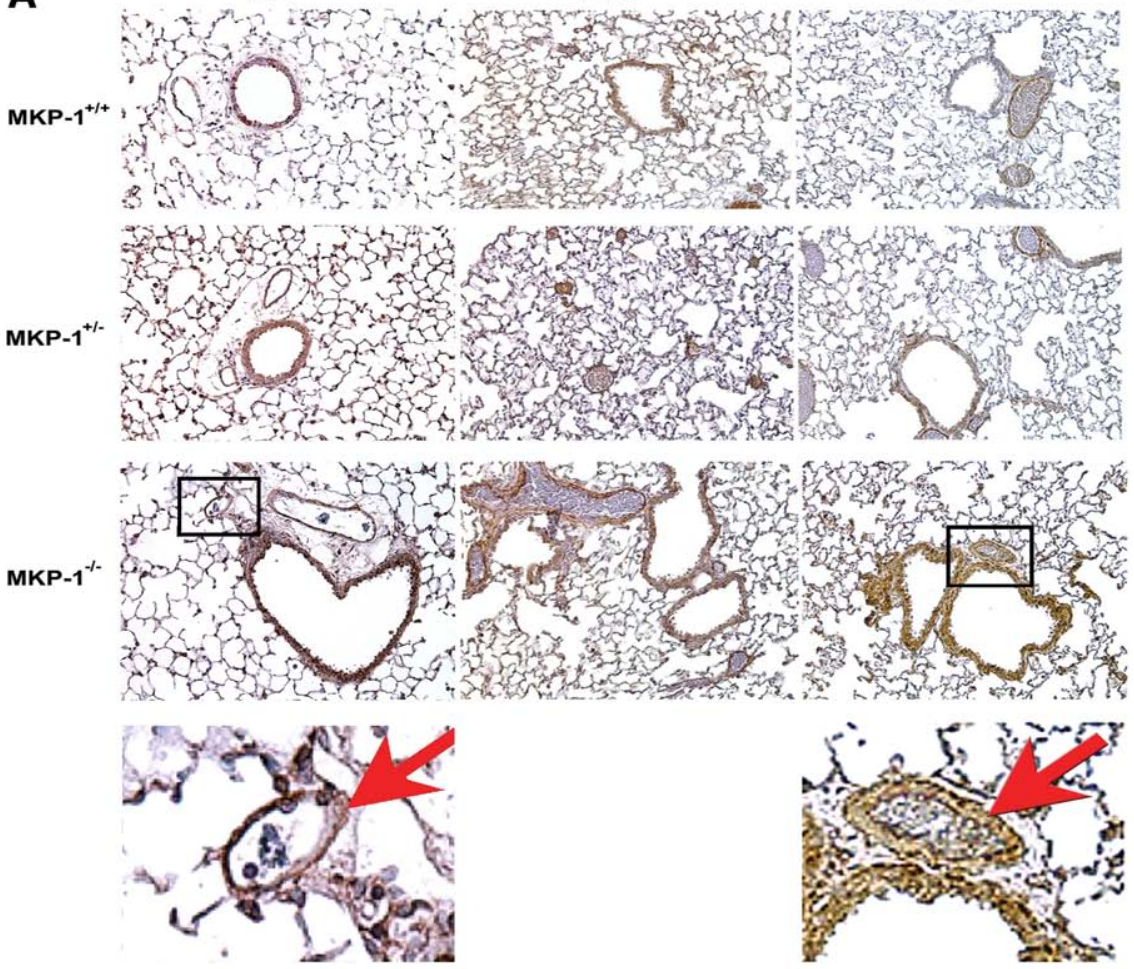

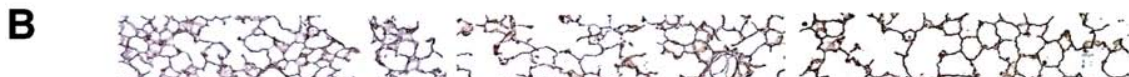

\section{MKP-1 $^{+1+}$} s.

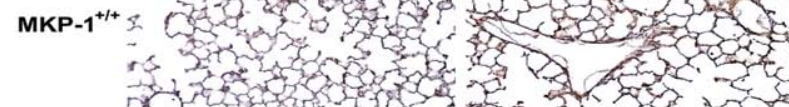
त.

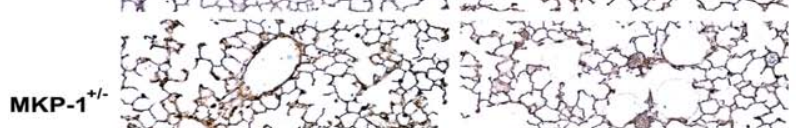

MKP-1

$\Rightarrow \rightarrow 3$ in

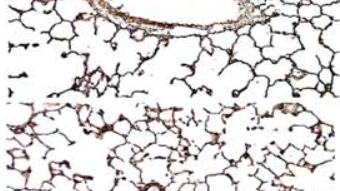

Figure 3. Deletion of MKP-1 affects the expression of $\alpha$ SMA and VEGF in the lung. A: $\alpha$ SMA expression is up-regulated in hypoxia-exposed MKP-1-deficient lung ( $n=3$ to 4 animals group). Insets identify areas from MKP-1 lung of SL and HYP groups that are magnified in the row below, with red arrows indicating the vessels positive for $\alpha$ SMA staining. B: VEGF expression is increased in hypoxic lung of wildtype mice ( $n=3$ to 4 animals/group). Original magnification $\times 400 ; \times 1600$ (insets) 
any altitude condition (Figure 2A). We did not detect any differences in the staining patterns of phosphoJNK $1 / 2$ in lung of all three genotypes (data not shown).

In SL-exposed lung of all three genotypes, either minimal or no positive reaction was observed with antibody against phosphoP38 MAP kinase (Figure 2B). However, activated p38 MAP kinase was detected by immunoperoxidase staining in DA-exposed lung of all genotypes (Figure 2B). The strongest signal for phosphoP38 MAP kinase was observed in HYP-exposed lung (Figure 2B). MKP-1 ${ }^{-1-}$ lung had maximal levels of p38 MAP kinase phosphorylation in the vascular wall, alveoli, and airways in response to chronic hypoxia exposure (Figure 2B). Interestingly, phosphorylated p38 MAP kinase might be localized in the intimal endothelial cells of the vascular wall in lung of MKP-1 $1^{-1-}$ mice (Figure 2B, arrow in inset). Taken together, these data suggest that activation of ERK1/2 (SL) and p38 MAP kinase (HYP) is greatest in lung of $\mathrm{MKP}_{-1} 1^{-1-}$ mice.

\section{MKP-1 $1^{-1-}$ Mice Have Altered Expression of $\alpha S M A$ and VEGF in the Lung}

To examine the role of MKP-1 in the development and maintenance of vessels, lung sections were stained with anti- $\alpha$ SMA and anti-VEGF antibodies. Mouse lung of all three genotypes expressed $\alpha$ SMA under all three altitude conditions. However, MKP $-1^{-1-}$ mice had greatest levels of $\alpha \mathrm{SMA}$ expression in lung at both DA and HYP conditions (Figure 3A). $\alpha$ SMA was expressed in the vessels, airways, and interstitial cells of MKP-1deficient lung (Figure 3A). Exaggerated $\alpha$ SMA expression in the vascular wall of MKP-1-null lung (Figure 3A, arrows in insets, SL and HYP groups) suggests that chronic exposure to hypoxia might trigger structural remodeling of the wall.

We then evaluated lung vasculature of these mice by VEGF immunostaining (Figure 3B). There were no striking differences in the staining patterns of VEGF in lung of $\mathrm{MKP}-1^{+/-}$and $\mathrm{MKP}-1^{-1-}$ mice across the three altitude conditions examined (Figure 3B). However, VEGF levels were up-regulated in HYP-exposed MKP$1^{+/+}$lung, because the staining intensity was enhanced (Figure 3B). Therefore, these data suggest that $\alpha S M A$ and VEGF expression in the lung might be affected by the absence of MKP-1 expression.

\section{Hypoxia Induces Reduction in and Remodeling

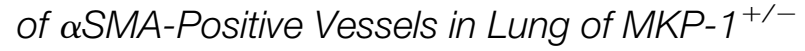 and $M K P-1^{-/-}$Mice}

We then counted $\alpha$ SMA-positive vessels ( $<50 \mu \mathrm{m}$ OD) and alveoli in $\alpha \mathrm{SMA}$-stained lung sections. In MKP$1^{+/+}$lung, vessel numbers were markedly decreased in the DA and HYP groups, compared with those of the $S L$ condition (Figure 4A). There was a trend toward an
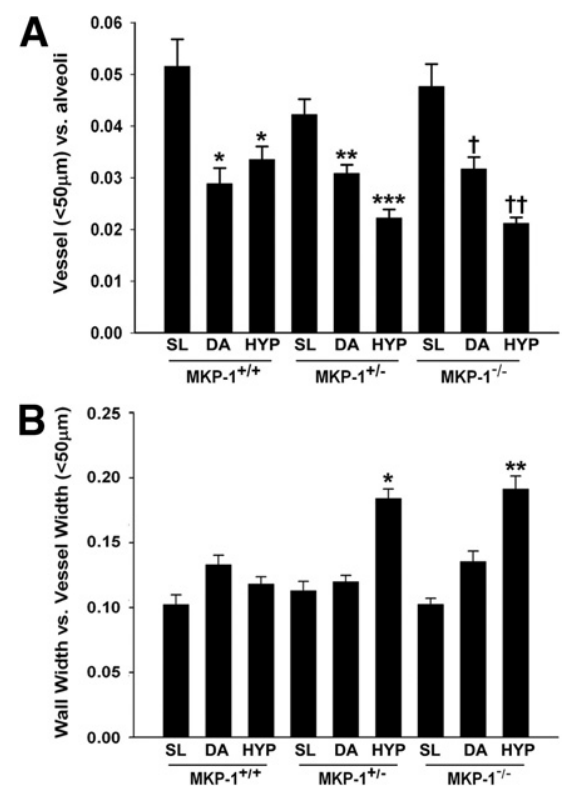

Figure 4. Hypoxia induces loss and structural remodeling of $\alpha$ SMA-positive vessels $(<50 \mu \mathrm{m}$ OD) in MKP-1-deficient lung. A: $\alpha$ SMA-positive vessel densities are markedly decreased in hypoxic lung of $\mathrm{MKP}_{-} 1^{+/-}$and MKP$1^{-1-}$ mice. Data reported as mean \pm SEM, 3 to 5 animals per group. ${ }^{*} P<$ 0.001 compared with SL-exposed MKP- $1^{+/+}$lung. *** $P<0.001$ compared with SL-exposed MKP-1 ${ }^{+/-}$lung. ${ }^{* * * *} P<0.01$ compared with SL- and DAexposed $\mathrm{MKP}-1^{+/-}$mice and HYP-exposed MKP- $1^{+/+}$mice. ${ }^{\dagger} P<0.001$ compared with SL-exposed MKP- $1^{-/-}$mice. ${ }^{\dagger t} P<0.05$ compared with SLand DA-exposed MKP-1 ${ }^{-/-}$mice and HYP-exposed MKP- $1^{+/+}$mice. $n=3$ to 5 animals/group; 5 to 12 vessels in each lung/animal were counted. B: Hypoxia induces structural remodeling of the vascular wall (wall width to vessel width ratio) in lung of $\mathrm{MKP}-1^{+/-}$and $\mathrm{MKP}-1^{-/-}$mice. ${ }^{*} P<0.001$ compared with SL- and DA-exposed MKP- $1^{+/-}$and HYP-exposed MKP- $1^{+/+}$ mice. ${ }^{* * *} P<0.001$ compared with SL- and DA-exposed MKP- ${ }^{-/-}$and HYP-exposed MKP- $1^{+/+}$mice. $n=3$ to 5 animals/group; at least 50 vessels/ animal were analyzed.

increase in the vessel/alveoli ratio in hypoxic lung of these mice, compared with those under DA conditions (Figure 4A); however, the difference was not statistically significant. In the case of MKP-1 $1^{+-}$and MKP$1^{-1-}$ mice, the vessel/alveoli ratio was sequentially reduced with increasing altitude throughout the lung (Figure 4A). In MKP-1-deficient lung, vessel densities were greatest under SL conditions and least under HYP conditions (Figure 4A), suggesting that MKP-1 might be an important regulator of the maintenance of vessel density in hypoxic lung.

Structural remodeling of the vessels $(<50 \mu \mathrm{m}$ OD) was evaluated by measuring the ratio of vessel wall width to vessel diameter. Altitude did not have any effect on the vascular wall thickness in the lung of wild-type mice. However, the ratio was markedly increased in HYP-exposed lung of MKP-1 ${ }^{+/-}$and MKP$1^{-1-}$ mice, indicating that the vessel wall underwent relative thickening with respect to the overall vessel diameter (Figure 4B). Remodeled vessels were distributed throughout the lungs of MKP-1-deficient mice. Taken together, these data suggest that MKP-1 is a crucial phosphatase for the development and maintenance of vessels $(<50 \mu \mathrm{m} \mathrm{OD})$ in the lung under hypoxic conditions. 


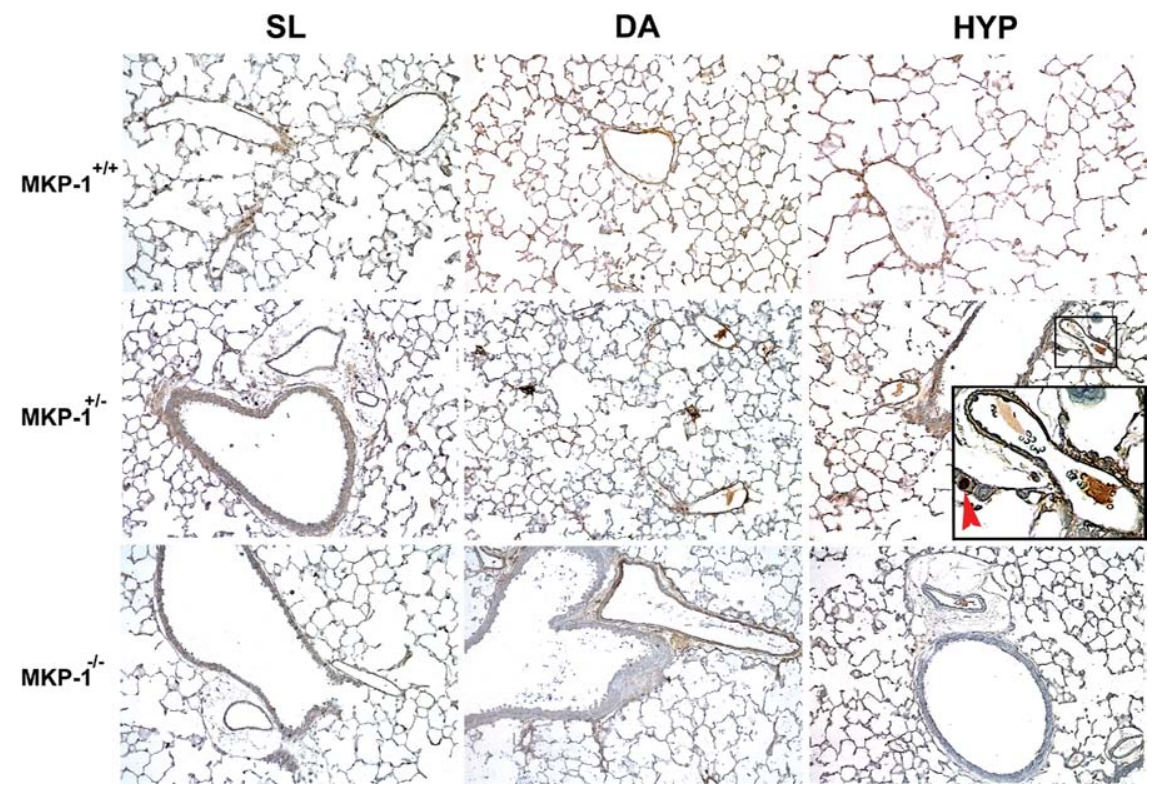

Figure 5. Ki-67-positive cells are most abundant in hypoxic lung of MKP-1 $1^{+/+}$mice. Representative images of lung from $\mathrm{MKP}^{+} 1^{++}, \mathrm{MKP}-$ $1^{+/-}$, and $\mathrm{MKP}-1^{-/-}$mice exposed to SL, DA and HYP for 6 weeks ( $n=3$ animals/group). In the MKP- $1^{+/-}$lung section of HYP group, the small box marks an example of Ki-67-positive nuclei, magnified in the inset; the red arrow points to a Ki-67-positive nucleus. Original magnification $\times 400 ; \times 1200$ (inset)

\section{Ki-67 Labeling Is Greatest in Hypoxia-Exposed Lung of Wild-Type Mice}

To evaluate whether vascular wall remodeling in hypoxic $\mathrm{MKP}_{-} 1^{+1-}$ and $\mathrm{MKP}_{-} 1^{-1-}$ lung is due to cell proliferation, lung sections were stained with antibody against Ki-67, a proliferative marker. Ki-67-positive vessels were not detectable in SL- or in DA-exposed lung of all three genotypes (Figure 5); in the HYPexposed lung, however, Ki-67 immunoreactivity became apparent. Hypoxia-exposed MKP-1+/+ lung had the highest number of Ki-67-positive cells, compared with those of $\mathrm{MKP}-1^{+/-}$and $\mathrm{MKP}_{-} 1^{-1-}$ animals (Figure $5)$. These data suggest that, at 6 weeks of hypoxia exposure, cell replication might not be present in the remodeled vessels of MKP-1-deficient lung.

\section{Hypoxia-Induced Activation of MAP Kinases Is Highest in MKP-1 ${ }^{-1-}$ MEFs}

To examine the mechanisms involved in hypoxia-induced modification of vasculature in mouse lung lacking MKP-1 expression, MEFs of all three genotypes were isolated and cultured. Hypoxia-stimulated activation of MAP kinases was evaluated in these MEFs using antibodies against phosphoMAP kinases. In MKP $-1^{+/+}$ cells, ERK1/2 were maximally (twofold) phosphorylated at 30 minutes of hypoxia exposure. ERK1/2 dephosphorylation occurred by the 60 minutes time point and was maintained up to 120 minutes of hypoxia exposure in wild-type cells (Figure 6A). Basal levels of phosphorylated ERK1, as evidenced by detection of a strong upper band on an immunoblot, were higher in MKP$1^{+/-}$MEFs than in MKP $-1^{+/+}$and MKP $-1^{-1-}$ cells (Figure $6 \mathrm{~A}$ ). There was no statistically significant increase in ERK1/2 activation in hypoxia-exposed $\mathrm{MKP}_{-1}{ }^{+/-}$ cells (Figure 6A). However, ERK1/2 phosphorylation was up-regulated fivefold in $\mathrm{MKP}-1^{-1-} \mathrm{MEFs}$ at 30 minutes of hypoxia exposure, which represents a time point of maximal ERK1/2 activation in response to hypoxic stimulation observed across the three genotypes (Figure 6A). Furthermore, ERK1/2 phosphorylation persisted in hypoxia-exposed MKP-1-null cells, albeit at lower levels, with threefold and twofold activation at 60 and 120 minutes, respectively (Figure 6A). Thus, MKP$1^{-1-}$ MEFs acquire highest and sustained ERK1/2 activation in response to hypoxic stimulation.

To develop a MAP kinase activation profile of hypoxic MEFs, we next evaluated JNK1/2 and p38 MAP kinase activation. Basal levels of phosphorylated JNK1/2 were high and not affected by hypoxia across all three cell types (Figure 6B). In the case of p38 MAP kinase, the basal phosphorylation levels were again considerably high in cells of all three genotypes (Figure $6 \mathrm{C}$ ). There was an increase in p38 MAP kinase phosphorylation in $\mathrm{MKP}-1^{+/+}$and $\mathrm{MKP}-1^{+/-}$cells after 30 minutes of hypoxia exposure; however, the up-regulation of phosphorylation levels was not statistically significant (Figure 6C). By contrast, p38 MAP kinase phosphorylation was augmented more than twofold in MKP $-1^{-1-}$ MEFs at 30 minutes of hypoxia exposure (Figure 6C). Taken together, these data suggest that response of MKP-1-deficient MEFs to hypoxia relies on phosphorylation of ERK1/2 and p38 MAP kinase, but not on JNK1/2 activation.

\section{VEGF Expression Is Blunted in Hypoxic MKP-1-Deficient MEFS}

To evaluate the levels of $\alpha$ SMA and VEGF, quiescent MEFs of all genotypes were exposed to either normoxia or hypoxia. The basal $\alpha$ SMA expression was noticeably higher in MKP-1-null cells than in the wild-type MEFs (Figure 7A). To evaluate the role of MAP kinases in $\alpha$ SMA expression, cells were stimulated with hypoxia either in the absence or in the presence of various 
A
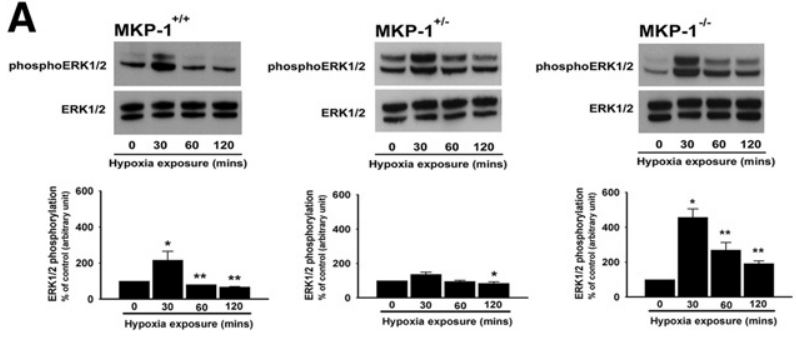

B
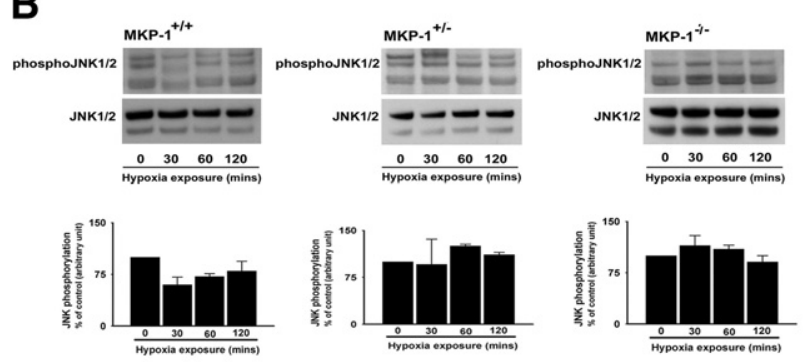

C

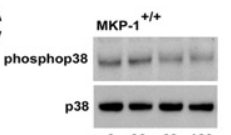
\begin{tabular}{llll}
$0 \quad 30 \quad 60 \quad 120$ \\
\hline
\end{tabular}
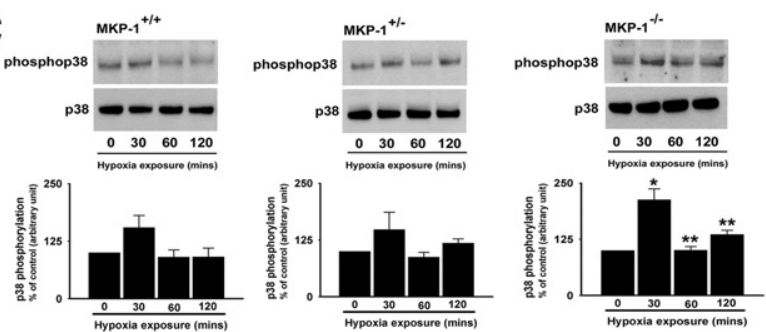

Figure 6. Hypoxic MKP-1 $1^{-/-}$MEFs exhibit maximal phosphorylation of ERK1/2 and p38 MAP kinase. A: ERK1/2 phosphorylation is sustained in hypoxic MKP-1-null MEFs ( $n=3$ to 4 cell populations each isolated from individual embryo). MKP- $1^{+/+}:{ }^{*} P<0.05$ compared with 0 hours. ${ }^{* *} P<0.05$ compared with 30 minutes. MKP- $1^{+/-}:{ }^{*} P<0.05$ compared with 30 minutes. MKP-1 ${ }^{-1-}:{ }^{*} P<0.01$ compared with 0 hours. ${ }^{* *} P<0.05$ compared with 30 minutes. B: MEFs have high basal JNK1/2 phosphorylation levels ( $n=3$ to 4 populations, each cultured from a different embryo). C: Only MKP-1-deficient MEFs have significant $\mathrm{p} 38$ activation in response to hypoxia exposure ( $n=3$ to 4 cell populations, each isolated from single embryo). MKP- $1^{-/-}:{ }^{*} P<0.05$ compared with 0 hours. ${ }^{* *} P<0.05$ compared with 30 minutes.

pharmacological antagonists of MAP kinase pathways. Only up on blockade of JNK1/2 with SP600125, hypoxia significantly up-regulated $\alpha S M A$ levels in MKP$1^{+/+}$MEFs suggesting that JNK $1 / 2$ might be a negative regulator of $\alpha S M A$ expression in these cells (Figure 7A). Neither hypoxia exposure nor blockade of MAP kinases with various inhibitors had any effect on $\alpha \mathrm{SMA}$ levels in MKP-1-deficient cells (Figure 7A). In MKP$1^{+/-}$MEFs, the findings regarding $\alpha S M A$ expression resembled those from the null cells. $\alpha$ SMA levels were unaffected by different inhibitors of MAP kinase pathways (data not shown). Therefore, $\alpha \mathrm{SMA}$ expression in MEFs might be regulated by MKP-1.

Because we hypothesized an effect of MKP-1 on VEGF expression, we next examined whether hypoxia stimulates increase in VEGF levels in the MEFs. Notably, eightfold up-regulation of VEGF levels was observed in hypoxia-exposed wild-type cells (Figure 7B). Blockade of ERK 1/2 and p38 MAP kinase with U0126 and SB203580, respectively, markedly blunted the increase in VEGF levels (Figure 7B). However, the greatest inhibitory effects on VEGF were found in hypoxic wild-type
MEFs in the presence of JNK $1 / 2$ attenuation by SP600125 (Figure 7B). In the case of MKP- $1^{+/}$MEFs, the basal levels were considerably greater $(300 \mathrm{pg} / \mathrm{ml})$ compared with that in wild-type cells $(31 \mathrm{pg} / \mathrm{ml})$ (Figure 7B). Hypoxia did not have any effect on VEGF expression in these cells, nor did different inhibitors of MAP kinase pathways (Figure 7B). MKP-1-null cells also possessed higher basal VEGF levels $(100 \mathrm{pg} / \mathrm{ml})$, compared with MKP $-1^{+/+}$MEFs (31 pg/ml) (Figure 7B). There was only a $70 \%$ increase in VEGF levels in hypoxic MKP $-1^{-1-}$ MEFs, a value that is markedly lower than for wild-type cells (Figure 7B). Furthermore, VEGF expression in hypoxiaexposed MKP $-1^{-1-}$ MEFs was reduced only in the presence of p38 inhibitor with SB203580 (Figure 7B).

\section{MKP-1 ${ }^{-1-}$ MEFs Lack Hypoxia-Stimulated Replication Responses}

To examine the role of MKP-1 in hypoxia-induced proliferative responses of the cells, growth-arrested MEFs were exposed to either normoxia or hypoxia for 72 hours. Increase in proliferation was observed in hypoxic MKP $-1^{+/+}$and MKP-1 $1^{+/-}$MEFs, but not in MKP$1^{-1-}$ MEFs (Figure 8). We then evaluated the effects of different MAP kinase inhibitors on proliferative responses of the cells. Hypoxia-induced replication in wild-type cells was reduced in the presence of ERK $1 / 2$ inhibition by U0126, JNK1/2 attenuation by SP600125, and p38 MAP kinase blockade by SB203580 (Figure
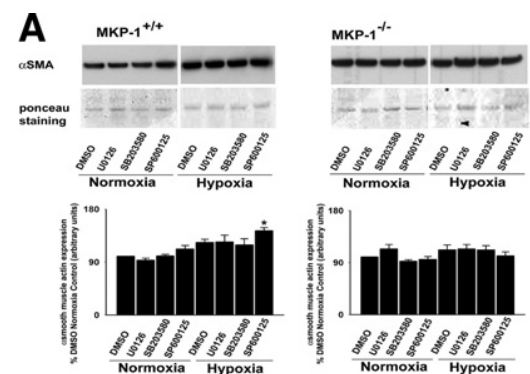

B
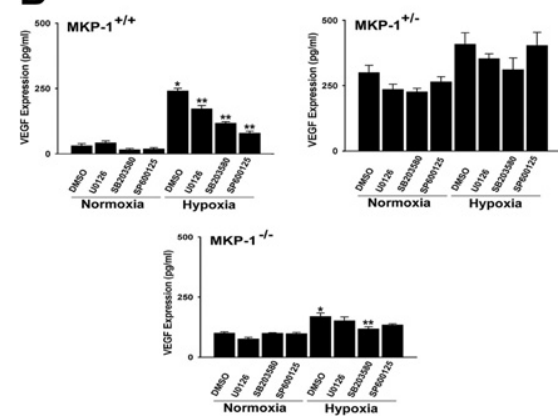

Figure 7. MKP- 1 depletion affects $\alpha$ SMA and VEGF expression in MEFs. A: Blockade of JNK pathways with SP600125 up-regulates $\alpha$ SMA expression in hypoxic MKP-1 ${ }^{+/+} \operatorname{MEFs}(n=3$ to 4 cell populations, each isolated from an individual embryo). ${ }^{*} P<0.05$ compared with the results of SP600125 treated cells under normoxic conditions. B: Hypoxia selectively up-regulates VEGF expression in MKP- $1^{+/+} \operatorname{MEFs}(n=3$ to 4 populations, each cultured from an individual embryo). MKP- $1^{+/+}:{ }^{*} P<0.001$ compared with normoxic dimethyl sulfoxide (DMSO). ${ }^{* *} P<0.001$ compared with hypoxic DMSO MKP-1 ${ }^{-/-}:{ }^{*} P<0.001$ compared with normoxic DMSO and ${ }^{* *} P<0.05$ compared with hypoxic DMSO. 

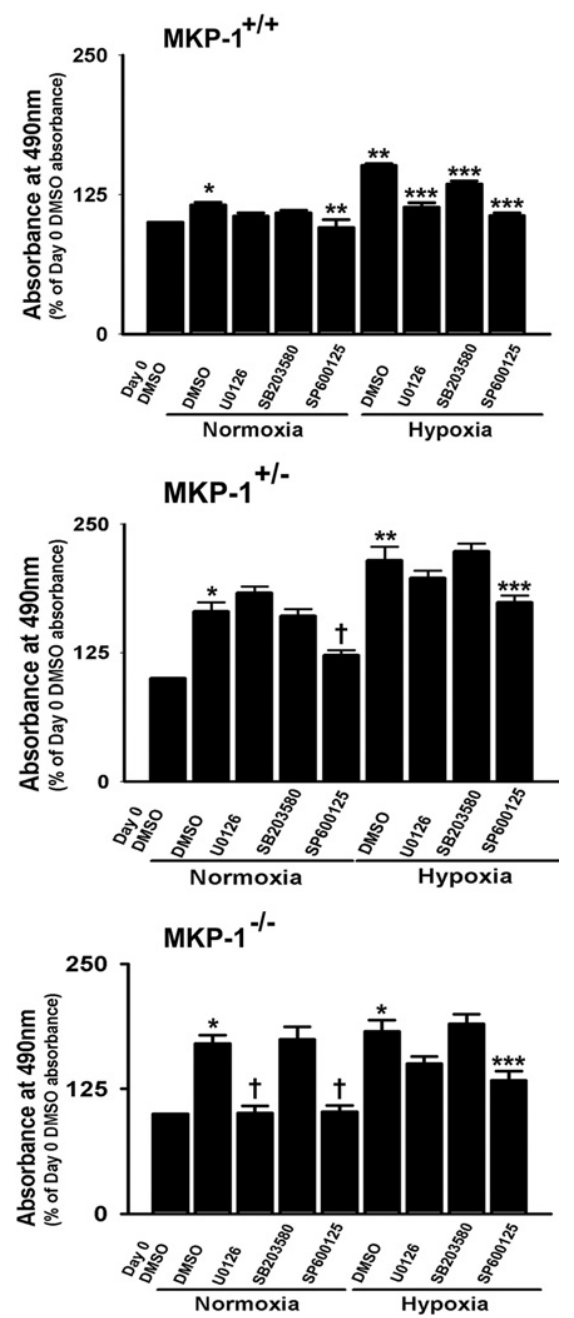

Figure 8. Hypoxia stimulates proliferation in MKP-1 $1^{+/+}$and $\mathrm{MKP}-1^{+/-}$but not in MKP-1 $1^{-/-}$MEFs. In MKP- $1^{+/+}$MEFs, hypoxia-induced replication was attenuated by inhibitors of MAP kinase pathway $(n=8$ replicate wells with 3 to 4 populations of MEFs, each isolated and cultured from individual embryo). ${ }^{*} P<0.05$ compared with absorbance value of day 0 DMSO for cells from all three genotypes. ${ }^{* *} P<0.001$ compared with day 3 DMSO under normoxic condition for MKP- $1^{+/+}$and MKP- $1^{+/-}$MEFs. ${ }^{* * * *} P<0.001$ compared with day 3 DMSO under hypoxic conditions for all three genotypes. ${ }^{\dagger} P<0.001$ compared with day 3 DMSO under normoxic conditions for $\mathrm{MKP}_{-} 1^{+/-}$and $\mathrm{MKP}^{-1}{ }^{-/-}$MEFs. Data reported as mean \pm SEM.

8). In the case of MKP-1 $1^{+/-}$and MKP-1 $1^{-1-}$ MEFs, replication rate was reduced only in the presence of JNK1/2 inhibition under hypoxic conditions (Figure 8). Our findings suggest that MEFs in the absence of MKP-1 expression might acquire dysregulated cell proliferation in response to hypoxic stimulation.

\section{Discussion}

In the present study, we found that MKP-1 functions as a key phosphatase in hypoxic lung through the regulation of VEGF levels and subsequent vessel density. We found that p38 MAP kinase, a substrate of MKP-1, is strongly activated in lung of MKP-1-deficient mice in response to chronic hypoxia exposure. Hypoxia also dramatically up-regulated $\alpha$ SMA expression in MKP-1- deficient lung. Most importantly, we demonstrated that chronic hypoxia exposure induces reduction and structural remodeling of $\alpha$ SMA-positive vessels $(<50$ $\mu \mathrm{m} \mathrm{OD)}$ in lung lacking the MKP-1 gene. Using isolated MKP-1-deficient MEFs, we also found that these cells have maximal and sustained hypoxia-induced activation of ERK $1 / 2$ and p38 MAP kinase. However, the cells have faulty hypoxia-stimulated VEGF expression, which might contribute to decrease in lung vasculature density of MKP-1-null mice in response to chronic hypoxia exposure. Our data strongly suggest that MKP-1 acts as an important phosphatase for the maintenance of vessels through the regulation of VEGF levels in hypoxic lung and thus represents a promising pharmacological target for the treatment of numerous vascular disorders.

MKP-1 belongs to a family of dual-specific phosphatases, able to dephosphorylate both threonine and tyrosine residues. Expression of MKP-1 is increased by cellular stressors such as hypoxia, UV light, oxidative stress, glucocorticoid stimulation, and heat shock. ${ }^{32-36}$ MKP-1-null mice exhibit enhanced ERK1/2, JNK1/2, and p38 MAP kinase activities and are resistant to diet-induced obesity, suggesting that this phosphatase might be a crucial regulator of body weight. ${ }^{14}$ The concept of MKP-1 as a weight-controlling phosphatase is in accord with our present findings, that chronic exposure to hypoxia interfered with weight gain in MKP-1-null mice (Figure 1B). Recently, Jin et $\mathrm{al}^{16}$ reported similar findings, that chronic hypoxia exposure (4 weeks) attenuated gain in body weight of adult MKP-1-null mice. It will be of broad interest to dissect the mechanisms involved in the regulatory role of MKP-1 in total body weight gain on challenge with chronic hypoxia exposure.

Studies using MKP-1-deficient mice have indicated a role for $\mathrm{MKP}-1$ as a negative regulator of Toll-like receptors, which are important in innate immunity. ${ }^{37}$ Although recent studies have demonstrated that MKP-1 plays an important role in ex vivo aortic angiogenesis ${ }^{9}$ and is a hypoxia-inducible phosphatase, ${ }^{4,32}$ the role of MKP-1 in the maintenance of vascular density in the lung, especially on hypoxic challenge, remains unknown. Our data regarding the loss and structural remodeling of vessels $(<50 \mu \mathrm{m} O D)$ in hypoxic MKP-1-null lung suggest that MKP-1 might act as a regulator of progression of various vascular diseases.

Hypoxia has been shown to promote angiogenesis via increase in MKP-1 expression. ${ }^{38}$ Treating sections of descending aorta from wild-type and MKP-1-null mice with VEGF or thrombin has revealed that endothelial cell sprouting is greatly reduced in MKP-1-null aortic sections, compared with control mice, confirming proangiogenic activity of MKP-1. ${ }^{9}$ In the present study, marked reduction in vessel densities in the hypoxic lung of MKP-1-deficient animals also strongly supports a critical regulatory role of this phosphatase as an angiogenic signaling mediator.

The classical paradigm in vessel formation implicates endothelial proliferation, migration, tube assembly, and remodeling as sequential steps. ${ }^{5}$ The loss of 
vessels could be due to alterations in endothelial cells and/or pericytes resulting in apoptosis. Indeed, vascular endothelial cells in lung of MKP-1-deficient mice have heightened p38 MAP kinase activation after 6 weeks of hypoxia exposure (Figure 2B). Given that p38 MAP kinase plays an important role in endothelial cell apoptosis, ${ }^{39}$ exaggerated p38 MAP kinase phosphorylation in endothelial cells might be one of the factors involved in vessel loss in MKP-1-deficient mice, a hypothesis that needs more rigorous examination in lung of MKP-1-null animals.

A role for phosphatases in either vessel formation or maintenance is also supported by other studies demonstrating that the phenotype of vascular endothelial protein tyrosine phosphatase knockout mice is embryonic lethal, with impaired vessel remodeling and maintenance. ${ }^{40,41}$ Although MKP-1-deficient mice appear to be normal under control conditions, our data strongly suggest that lungs of these mice have blunted response to hypoxia, due to the defective expression of the major angiogenic factor VEGF, as well as flaws in vessel formation or maintenance.

Decrease in vascularity has been shown to be involved in vascular remodeling. ${ }^{42}$ Endothelial cell apoptosis might contribute to vascular remodeling via release of secondary mediators leading to smooth muscle cell proliferation. ${ }^{43}$ In the present study, endothelial cell apoptosis might also be an important mechanism by which vascular remodeling develops in hypoxic $\mathrm{MKP}_{-} 1^{+/-}$and $\mathrm{MKP}_{-1} 1^{-1-}$ lung. However, proliferative index of vascular cells as evaluated by Ki-67 staining (Figure 5) was not higher in hypoxic MKP-1-deficient lung. In fact, Ki-67-positive nuclei were most numerous in hypoxic lung of wild-type mice. Greater proliferating cell nuclear antigen (PCNA) levels have been recently detected in lung of hypoxic (4 weeks exposure) MKP-1 $1^{-1-}$ mice than that in hypoxic wild-type mice. ${ }^{16}$ Because these PCNA levels have been evaluated by Western blot analysis in total lung homogenate, it is very difficult to localize hypoxia-stimulated enhanced proliferative responses in lung of MKP-1-deficient mice.

Duration of hypoxia exposure might also be an important determinant for the detection of hypoxia-induced replication rate in lung of MKP-1-deficient mice. We have future plans to examine the proliferative responses of vascular wall cells in MKP-1 null lungs more completely, by performing time-course studies of hypoxia-induced $\mathrm{BrdU}$ incorporation in the lungs of these mice. Our experiments with isolated MEFs demonstrated that cells lacking MKP-1 expression lose the ability to divide under hypoxic conditions. In contrast, hypoxia stimulates an increase in cell numbers in wildtype MEFs (Figure 8). Therefore, cell proliferation after 6 weeks of hypoxia exposure might not be a major determining factor for vascular remodeling in MKP-1deficient lung. Marked up-regulation of $\alpha$ SMA expression in MKP-1-deficient lung under hypoxic conditions (Figure 3A) suggests that smooth muscle cell hypertrophy could be involved in remodeling process of the vascular wall in the lung lacking MKP-1 expression.
VEGF is an important survival factor for endothelial cells and hypoxia up-regulates its expression. ${ }^{5,44} \mathrm{Al}$ though MKP-1 has been identified as a novel VEGFinducible gene, ${ }^{9,10}$ regulation of hypoxia-induced VEGF expression by MKP-1 remains unexplored. The present findings demonstrate that VEGF expression is tightly regulated by MKP-1 in hypoxic MEFs. Hypoxia induces marked up-regulation of VEGF expression in wild-type cells. However, MKP-1-null cells lack this response, suggesting that this phosphatase is an important mediator of hypoxia-induced VEGF expression. Another important point is that the increase in VEGF levels in hypoxic MKP $-1^{+/+}$MEFs is highly dependent on JNK1/2 activation; attenuation of JNK $1 / 2$ with SP600125 led to greatest reduction in VEGF expression in these cells, compared with ERK and p38 MAP kinase inhibitors (Figure 7B). In contrast, MKP-1-deficient cells do not require JNK1/2 activation for VEGF expression. Therefore, defective VEGF expression and the subsequent rarefaction of vascular structures, together with remodeling, suggest that lack of MKP-1 expression in endothelial cells might induce impaired responses in MKP-1-deficient mice challenged by hypoxia.

In the present study, hypoxia-induced sustained activation of ERK $1 / 2$ in isolated MKP-1 $1^{-1-}$ MEFs (Figure 6A) was not recapitulated in the in vivo phosphorylation pattern of this kinase (Figure 2A). Only MKP-1-deficient lung from the SL group had increased ERK1/2 phosphorylation. Discrepancies in the activation patterns might be due either to the extended in vivo experimental period or to over-expression of other members of MKP family. Six weeks of hypoxia exposure might be an exceedingly long time for the detection of ERK $1 / 2$ phosphorylation in the lung. However, in the case of p38 MAP kinase, we found hypoxia-stimulated sustained phosphorylation of the kinase, both in vitro and in vivo, suggesting that p38 MAP kinase activation might be a major signaling kinase in lung of MKP-1-deficient mice. The role of p38 MAP kinase-responsive genes in the physiological responses of MKP-1-null lung to hypoxia needs further evaluation.

MKP-1 likely acts as a signaling switch that attenuates the phosphorylation of p38 MAP kinase, JNK, and ERK, thus allowing the expression of genes necessary for VEGF production and vessel formation in the lung. Consequently, strategies specifically targeting MKP-1 may be of benefit in potentiating hypoxic signaling and enhancing the formation and maturation of neovessels in the context of therapeutic angiogenesis.

\section{Acknowledgments}

We thank Lexicon Pharmaceuticals, Inc., for providing MKP-1-null mice. We also thank Dr. Kurt Stenmark, Dr. Carlyne Cool (University of Colorado Denver), and Dr. James West (Vanderbilt University) for critical comments on the manuscript. 


\section{References}

1. Camps M, Nichols A, Arkinstall S: Dual specificity phosphatases: a gene family for control of MAP kinase function. FASEB J 2000, 14 $6-16$

2. Misra-Press A, Rim CS, Yao H, Roberson MS, Stork PJS: A novel mitogen-activated protein kinase phosphatase. Structure, expression and regulation. J Biol Chem 1995, 270:14587-14596

3. Zakkar M, Chaudhury H, Sandvik G, Enesa K, Luong LA, Cuhlmann S Mason JC, Krams R, Clark AR, Haskard DO, Evans PC: Increased endothelial mitogen-activated protein kinase phosphatase-1 expression suppresses proinflammatory activation at sites that are resistant to atherosclerosis. Circ Res 2008, 103:726-732

4. Seta KA, Kim R, Kim HW, Millhorn DE, Beitner-Johnson D: Hypoxiainduced regulation of MAPK phosphatase- 1 as identified by subtractive suppression hybridization and cDNA microarray analysis. J Biol Chem 2001, 276:44405-44412

5. Carmeliet P: Angiogenesis in health and disease. Nat Med 2003, 9:653-660

6. Tuder RM, Flook BE, Voelkel NF: Increased gene expression for VEGF and the VEGF receptors KDR/FIk and Flt in lungs exposed to acute or to chronic hypoxia. Modulation of gene expression by nitric oxide. $\mathrm{J}$ Clin Invest 1995, 95:1798-1807

7. Christou H, Yoshida A, Arthur V, Morita T, Kourembanas S: Increased vascular endothelial growth factor production in the lungs of rats with hypoxia-induced pulmonary hypertension. Am J Respir Cell Mol Biol 1998, 18:768-776

8. Marti HH, Risau W: Systemic hypoxia changes the organ-specific distribution of vascular endothelial growth factor and its receptors. Proc Natl Acad Sci USA1998, 95:15809-15814

9. Kinney CM, Chandrasekharan UM, Mavrakis L, DiCorleto PE: VEGF and thrombin induce MKP-1 through distinct signaling pathways: role for MKP-1 in endothelial cell migration. Am J Physiol Cell Physiol 2008, 294:C241-C250

10. Bellou S, Hink MA, Bagli E, Panopoulou E, Bastiaend PIH, Murphy C, Fotsis T: VEGF auto-regulates its proliferative and migratory ERK $1 / 2$ and p38 cascades by enhancing the expression of DUSP1 and DUSP5 phosphatases in endothelial cells. Am J Physiol Cell Physiol 2009, 297:C1477-C1489

11. Dorfman K, Carrasco D, Gruda M, Ryan C, Lira SA, Bravo R: Disruption of the erp/MKP-1 gene does not affect mouse development normal MAP kinase activity in ERP/MKP-1-deficient fibroblasts. Oncogene 1996, 13:925-931

12. Salojin KV, Owusu IB, Millerchip KA, Potter M, Platt KA, Oravecz T: Essential role of MAPK phosphatase-1 in the negative control of innate immune responses. J Immunol 2006, 176:1899-1907

13. Zhao Q, Wang X, Nelin LD, Yao Y, Matta R, Manson ME, Baliga RS, Meng $X$, Smith CV, Bauer JA, Chang CH, Liu Y: MAP kinase phosphatase-1 controls innate immune response and suppresses endotoxic shock. J Exp Med 2006, 203:131-140

14. Wu JJ, Roth RJ, Anderson EJ, Hong EG, Lee MK, Choi CS, Neufer PD, Shulman GI, Kim JK, Bennett AM: Mice lacking MAP kinase phosphatase-1 have enhanced MAP kinase activity and resistance to diet-induced obesity. Cell Metab 2006, 4:61-73

15. Carison J, Cui W, Zhang Q, Xu X, Mercan F, Bennett AM, Vignery A: Role of MKP-1 in osteoclasts and bone homeostasis. Am J Pathol 2009, 175:1564-1573

16. Jin Y, Calvert TJ, Chen B, Chicoine LG, Joshi M, Bauer JA, Liu Y, Nelin LD: Mice deficient in MKP-1 develop more severe pulmonary hypertension and greater lung protein levels of arginase in response to chronic hypoxia. Am J Physiol Heart Circ Physiol 2010, 298:H1518H1528

17. Nagaoka T, Gebb SA, Karoor V, Homma N, Morris KG, McMurtry IF, Oka M: Involvement of RhoA/Rho kinase signaling in pulmonary hypertension of the fawn-hooded rat. J Appl Physiol 2006, 100:9961002

18. Homma N, Nagaoka T, Morio Y, Ota H, Gebb SA, Karoor V, McMurtry IF, Oka M: Endothelin-1 and serotonin are involved in activation of RhoA/Rho kinase signaling in the chronically hypoxic hypertensive rat pulmonary circulation. J Cardiovasc Pharmacol 2007, 50:697-702

19. Thomas M, Docx C, Holmes AM, Beach S, Duggan N, England K, Leblanc C, Lebret C, Schindler F, Raza F, Walker C, Crosby A, Davies RJ, Morrell NW, Budd DC: Activin-Like Kinase 5 (ALK5) mediates abnormal proliferation of vascular smooth muscle cells from patients with familial pulmonary arterial hypertension and is involved in the progression of experimental pulmonary arterial hypertension induced by monocrotaline. Am J Pathol 2009, 174:380-389

20. Dempsey EC, Wick MJ, Karoor V, Barr EJ, Tallman DW, Wehling CA Walchak SJ, Laudi S, Le M, Oka M, Majka S, Cool CD, Fagan KA Klemm DJ, Hersh LB, Gerard NP, Gerard C, Miller YE: Neprilysin null mice develop exaggerated pulmonary vascular remodeling in response to chronic hypoxia. Am J Pathol 2009, 174:782-796

21. Liu W, Liang Q, Balzar S, Wenzel S, Gorska M, Alam R: Cell-specific activation profile of extracellular signal-regulated kinase 1/2, Jun $\mathrm{N}$-terminal kinase, and p38 mitogen-activated protein kinases in asthmatic airways. J Allergy Clin Immunol 2008, 121:893-902.e2

22. Gesslein B, Håkansson G, Carpio R, Gustafsson L, Perez MT, Malmsjö M: Mitogen-activated protein kinases in the porcine retinal arteries and neuroretina following retinal ischemia-reperfusion. Mol Vis 2010, 16:392-407

23. Li J, Kuzin I, Moshkani S, Proulx ST, Xing L, Skrombolas D, Dunn R, Sanz I, Schwarz EM, Bottaro A: Expanded $C D 23^{+} / C D 21^{\text {hi }}$ B cells in inflamed lymph nodes are associated with the onset of inflammatoryerosive arthritis in TNF-transgenic mice and are targets of anti-CD20 therapy. J Immunol 2010, 184:6142-6150

24. Khan SJ, Pham S, Wei Y, Mateo D, St-Pierre M, Fletcher TM, VazquezPadron RI: Stress-induced senescence exaggerates postinjury neointimal formation in the old vasculature. Am J Physiol Heart Circ Physiol 2010, 298:H66-H74

25. Giatromanolaki A, Koukourakis MI, Sivridis E, Turley H, Wykoff CC Gatter KC, Harris AL: DEC1 (STRA13) protein expression relates to hypoxia-inducible factor 1-alpha and carbonic anhydrase-9 overexpression in non-small cell lung cancer. J Pathol 2003, 200:222-228

26. Das M, Burns N, Wilson SJ, Zawada WM, Stenmark KR: Hypoxia exposure induces the emergence of fibroblasts lacking replication repressor signals of PKCzeta in the pulmonary artery adventitia. Cardiovasc Res 2008, 78:440-448

27. Morrell NW, Atochina EN, Morris KG, Danilov SM, Stenmark KR Angiotensin converting enzyme expression is increased in small pulmonary arteries of rats with hypoxia-induced pulmonary hypertension [Erratum appeared in J Clin Invest 1996, 97:271]. J Clin Invest 1995 96:1823-1833

28. Long L, MacLean MR, Jeffery TK, Morecroft I, Yang X, Rudarakanchana N, Southwood M, James V, Trembath RC, Morrell NW: Serotonin increases susceptibility to pulmonary hypertension in BMPR2 deficient mice. Circ Res 2006, 98:818-827

29. Crosby A, Jones FM, Southwood M, Stewart S, Schermuly R, Butrous G, Dunne DW, Morrell NW: Pulmonary vascular remodeling correlates with lung eggs and cytokines in murine schistosomiasis. Am J Respir Crit Care Med 2010, 181:279-288

30. Das M, Bouchey DM, Moore MJ, Hopkins DC, Nemenoff RA, Stenmark KR: Hypoxia-induced proliferative response of vascular adventitial fibroblasts is dependent on G-protein-mediated activation of mitogen-activated protein kinases. J Biol Chem 2001, 276:1563115640

31. Short MD, Fox SM, Lam CF, Stenmark KR, Das M: Protein kinase Czeta attenuates hypoxia-induced proliferation of fibroblasts by regulating MAP kinase phosphatase-1 expression. Mol Biol Cell 2006 17:1995-2008

32. Laderoute KR, Mendonca HL, Calaoagan JM, Knapp AM, Giaccia AJ Stork PJ: Mitogen-activated protein kinase phosphatase-1 (MKP-1) expression is induced by low oxygen conditions found in solid tumor microenvironments. A candidate MKP for the inactivation of hypoxia-inducible stress-activated protein kinase/c-Jun N-termina protein kinase activity. J Biol Chem 1999, 274:12890-12897

33. Liu Y, Gorospe M, Yang C, Holbrook NJ: Role of mitogen-activated protein kinase phosphatase during the cellular response to genotoxic stress. Inhibition of c-Jun N-terminal kinase activity and AP-1 dependent gene activation. J Biol Chem 1995, 270:8377-8380

34. Keyse SM, Emslie EA: Oxidative stress and heat shock induce a human gene encoding a protein-tyrosine phosphatase. Nature 1992 359:644-647

35. Kassel O, Sancono A, Krätzschmar J, Kreft B, Stassen M, Cato AC Glucocorticoids inhibit MAP kinase via increased expression and decreased degradation of MKP-1. EMBO J 2001, 20:7108-7116 
36. Wong HR, Dunsmore KE, Page K, Shanley TP: Heat shock-mediated regulation of MKP-1. Am J Physiol Cell Physiol 2005, 289:C1152C1158

37. Chi H, Flavell RA: Acetylation of MKP-1 and the control of inflammation. Sci Signal 2008, 41:pe44

38. Boutros T, Chevet E, Metrakos P: Mitogen-activated protein (MAP) kinases/MAP kinase phosphatase regulation: roles in cell growth, death and cancer. Pharmacol Rev 2008, 60:261-310

39. McMullen ME, Bryant PW, Glembotski CC, Vincent PA, Pumiglia $\mathrm{KM}$ : Activation of p38 has opposing effects on the proliferation and migration of endothelial cells. J Biol Chem 2005, 280:20995-21003

40. Baumer S, Keller L, Holtman A, Funke R, August B, Gamp A, Wolburg H, Wolburg-Buchholz K, Deutsch U, Vestweber D: Vascular endothelial cell-specific phosphotyrosine phosphatase (VEPTP) activity is required for blood vessel development. Blood 2006, 107:4754-4762
41. Dominguez MG, Hughes VC, Pan L, Simmons M, Daly C, Anderson K, Noguera-Troise I, Murphy AJ, Valenzuela DM, Davis S, Thurston G, Yancopoulos GD, Gale NW: Vascular endothelial tyrosine phosphatase (VE-PTP)-null mice undergo vasculogenesis but die embryonically because of defects in angiogenesis. Proc Natl Acad Sci USA 2007, 104:3243-3248

42. Howell K, Ooi H, Preston R, McLoughlin P: Structural basis of hypoxic pulmonary hypertension: the modifying effect of chronic hypercapnia. Exp Physiol 2003, 89:66-72

43. Sakao S, Taraseviciene-Stewart L, Wood K, Cool CD, Voelkel NF: Apoptosis of pulmonary microvascular endothelial cells stimulates vascular smooth muscle cell growth. Am J Physiol Lung Cell Mol Physiol 2006, 291:L362-L368

44. Voelkel NF, Hoeper M, Maloney J, Tuder RM: Vascular endothelial growth factor in pulmonary hypertension. Ann N Y Acad Sci 1996 796:186-193 